\title{
Triviality of the generalized Lau product associated to a Banach algebra homomorphism
}

\author{
Yemon Choi
}

December 2, 2015

\begin{abstract}
Several papers have, as their raison d'être, the exploration of the generalized Lau product associated to a homomorphism $T: B \rightarrow A$ of Banach algebras. In this short note, we demonstrate that the generalized Lau product is isomorphic as a Banach algebra to the usual direct product $A \oplus B$. We also correct some misleading claims made about the relationship between this generalized Lau product, and an older construction of Monfared (Studia Mathematica, 2007).
\end{abstract}

MSc 2010 classification: $46 \mathrm{H} 20$

\section{The generalized Lau product}

Motivated by a construction of M. S. Monfared [10], several authors have in recent years written papers on a certain construction, which manufactures a Banach algebra $A \times_{T} B$ given a pair of Banach algebras $(A, B)$ and a continuous algebra homomorphism $T: B \rightarrow A$.

Definition 1. The underlying Banach space of $A \times_{T} B$ is the usual product/sum $A \oplus B$; multiplication is defined by the following rule:

$$
\left(a_{1}, b_{1}\right) \bullet_{T}\left(a_{2}, b_{2}\right)=\left(a_{1} a_{2}+T\left(b_{1}\right) a_{2}+a_{1} T\left(b_{2}\right), b_{1} b_{2}\right)
$$

It is clear that $\bullet_{T}$ is continuous, and easily verified by hand that it is associative (although this will also follow from the proof of Proposition 3). By renorming if necessary to obtain a submultiplicative norm, one obtains a Banach algebra $A \times_{T} B$. 
The construction in Definition 1 has gone by various names: "generalized Lau product"; "the T-Lau product"; or "the Lau product defined by a Banach algebra morphism". This last phrase is the one used in [3], which appears to be the earliest occurrence of this construction. Since [3] there have been several papers on the theme of the "generalized Lau product", by various authors: see [1], [2], 4], [5], [6], 7], 8], 9], 11], 12], 13].

Remark 2. In [3] it is claimed that this construction extends that of [10], and that it might have some bearing on extensions of $C^{*}$-algebras in the sense of BDF theory. The second claim is not given clear justfication in the article; and the first claim is misleading, to say the least, as will be explained in Section 2).

Unfortunately, the following elementary observation casts doubt on the whole enterprise. We leave the proof to the reader.

Proposition 3. Let $A \oplus_{\text {alg }} B$ denote the usual sum of Banach algebras, i.e. we equip the Banach spaces $A \oplus B$ with co-ordinatewise product

$$
\left(a_{1}, b_{1}\right) \cdot\left(a_{2}, b_{2}\right)=\left(a_{1} a_{2}, b_{1} b_{2}\right)
$$

Define $\phi: A \times_{T} B \rightarrow A \oplus_{\text {alg }} B$ by $\phi(a, b)=(a+T(b), b)$. Then $\phi$ is continuous, linear and bijective; and $\phi\left((a, b) \bullet_{T}(c, d)\right)=\phi(a, b) \cdot \phi(c, d)$. In particular, $A \times_{T} B$ is isomorphic as a Banach algebra to $A \oplus_{\text {alg }} B$.

Remark 4. The proof of Proposition 3 was found when the present author was reading the preprint [13], in particular the proof of Proposition 4.1 in that paper. It seems that the authors of [13] noticed something very similar to the isomorphism of Proposition 3, but only in a restricted setting. Similarly, it is observed just after Theorem 4.2 of [6] that there is an isomorphism $A \times_{T} B \rightarrow A \oplus_{\text {alg }} B$, but the authors of [6] only state this in the case where $A$ and $B$ are commutative and semisimple, and do not mention that Proposition 3 applies in full generality.

Hopefully, any future attempts to study the generalized Lau product, as defined in Definition 1, will bear in mind that $A \times_{T} B \cong A \oplus_{\text {alg }} B$ regardless of the choice of $T$. It is a basic theme, when defining a property of Banach algebras, to see how it behaves under forming binary sums/products of algebras. For many of the properties considered in the items of the bibliography, stability of such properties under forming binary sums/products is either known or refuted by old work. 


\section{Comparison with an older construction}

It is claimed in [3] that the construction presented there extends the one studied by Monfared in [10]. If this were true, then Monfared's construction would be a special case of Definition 1, and hence would be trivial for the same reason.

In fact, it is not true that Monfared's construction is a special case of Definition 1, In this section we shall briefly explain why.

Definition 5 (Monfared, [10]). Let $A$ and $B$ be Banach algebras and let $\varphi$ : $B \rightarrow \mathbb{C}$ be a character, i.e. a non-zero homomorphism. The Lau product of $A$ and $B$ with respect to $\varphi$ is defined to be the Banach algebra whose underlying Banach space is $A \oplus B$, equipped with the multiplication operation

$$
\left(a_{1}, b_{1}\right) \bullet \varphi\left(a_{2}, b_{2}\right)=\left(a_{1} a_{2}+\varphi\left(b_{1}\right) a_{2}+a_{1} \varphi\left(b_{2}\right), b_{1} b_{2}\right) .
$$

As observed by Bhatt and Dabhi, it is immediate that when $A$ has an identity element $e_{A}$ and $\varphi$ is a character on $B$, we may define a continuous homomorphism $T: B \rightarrow A$ by $T(a)=\varphi(b) e_{A}$. If we do this, then the Lau product of $A$ and $B$ with respect to $\varphi$ does indeed coincide with $A \times_{T} B$, and hence by Proposition 3 it is isomorphic to the usual direct product of algebras $A \oplus_{\text {alg }} B$.

What seems to have gone unremarked in [3] is that when $A$ does not have an identity element, Definition [5 is no longer a special case of Definition 1 . Indeed, in general the Lau product of $A$ and $B$ is not isomorphic as an algebra to $A \oplus_{\text {alg }} B$. This is not a new observation - it is implicit in [10] - but for sake of completeness we shall give an example to illustrate this.

Example 6. Let $A$ be an arbitrary Banach algebra. Take $B=\mathbb{C}$ and let id denote the identity map on $\mathbb{C}$. It was observed in 10 (and it is also clear from Definition (5) that the corresponding Lau product of $A$ and $B$ is isomorphic to $A^{\sharp}$, the usual unitization of $A$. Observe that $A \oplus_{\text {alg }} \mathbb{C}$ has an identity element if and only if $A$ does. Hence, if $A=c_{0}(\mathbb{N})$ (for example), then $A \oplus_{\text {alg }} \mathbb{C}$ cannot be isomorphic to $A^{\sharp}$.

A minor variation on the proof of Proposition 3 shows that given $A, B, \varphi$ as in Definition 5, there is a natural way to identify $A \times{ }_{\varphi} B$ with a closed, codimension-one subalgebra (not an ideal, in general!) of $A^{\sharp} \oplus_{\text {alg }} B$. Given that there continue to be papers exploring the Lau product (as in Definition (5), it therefore seems worthwhile to record the following consequence, whose proof we omit since it is straightforward. 
Proposition 7. Let $Q$ be a property of Banach algebras which is preserved by all isomorphisms of Banach algebras (not just the isometric ones). Suppose also that:

- if $A$ has the property $Q$, then so does $A^{\sharp}$;

- if $A_{1}$ and $A_{2}$ have the property $Q$, then so does $A_{1} \oplus_{\text {alg }} A_{2}$;

- if $A$ has the property $Q$ and $B$ is a closed subalgebra of $A$ with finite codimension, then $B$ has the property $Q$.

Then the property $Q$ is preserved by taking Lau products in the sense of Definition 5 .

Note that two examples of such properties are: Arens regularity; and being isomorphic to a closed subalgebra of $\mathcal{B}(H)$ for some Hilbert space $H$.

\section{Acknowledgements}

The author thanks M. Nemati for pointing out the reference [6], and thanks L. Molnar for helpful exchanges. He also thanks the referee for a suggestion which improved Example 6 .

\section{References}

[1] F. Abtahi and A. Ghafarpanah, A note on cyclic amenability of the Lau product of Banach algebras defined by a Banach algebra morphism, Bull. Aust. Math. Soc., 92 (2015), pp. 282-289.

[2] F. Abtahi, A. Ghafarpanah, and A. Rejali, Biprojectivity and biflatness of Lau product of Banach algebras defined by a Banach algebra morphism, Bull. Aust. Math. Soc., 91 (2015), pp. 134-144.

[3] S. J. Bhatt and P. A. Dabhi, Arens regularity and amenability of Lau product of Banach algebras defined by a Banach algebra morphism, Bull. Aust. Math. Soc., 87 (2013), pp. 195-206.

[4] —- Arens regularity and amenability of Lau product of Banach algebras defined by a Banach algebra morphism, Bull. Aust. Math. Soc., 87 (2013), pp. 195-206. 
[5] P. A. Dabhi, A. Jabbari, and K. Haghnejad Azar, Some notes on amenability and weak amenability of Lau product of Banach algebras defined by a Banach algebra morphism, Acta Math. Sin. (Engl. Ser.), 31 (2015), pp. 1461-1474.

[6] H. Javanshiri and M. Nemati, The multiplier algebra and BSEfunctions for certain product of Banach algebras. Preprint, see arXiv 1509.00895 .

[7] — On a certain product of Banach algebras and some of its properties, Proc. Rom. Acad. Ser. A Math. Phys. Tech. Sci. Inf. Sci., 15 (2014), pp. 219-227.

[8] A. R. Khoddami, n-weak amenability of T-Lau product of Banach algebras, Chamchuri J. Math., 5 (2013), pp. 57-65.

[9] —, On Banach algebras induced by a certain product, Chamchuri J. Math., 6 (2014), pp. 89-96.

[10] M. S. Monfared, On certain products of Banach algebras with applications to harmonic analysis, Studia Math., 178 (2007), pp. 277-294.

[11] M. Nemati And H. Javanshiri, Some homological and cohomological notions on T-Lau product of Banach algebras, Banach J. Math. Anal., 9 (2015), pp. 183-195.

[12] A. Pourabbas And N. Razi, Cohomological characterization of TLau product algebras. Preprint, see arxiv 1509.01933.

[13] — Some homological properties of T-Lau product algebra. Preprint, see arXiv 1411.0112.

Department of Mathematics and Statistics

Lancaster University

Lancaster, LA1 4YF

United Kingdom

Email: y.choi.97@cantab.net 